\title{
LOCULATED PYOTHORAX, PLEURAL THICKENING DUE TO ACQUIRED BOCHDALEK HERNIA AND BARIUM INGESTION- A RARE CASE.
}

\author{
G. Vijayalaxmi, S. Kartika Balaji, K. M. Karthik Raj, R. Pratap, Raju Deshmi
}

1. Professor. Department of Anaesthesiology, GSL Medical College, Rajahmundry.

2. Senior Resident. Department of Anaesthesiology, GSL Medical College, Rajahmundry.

3. Senior Resident. Department of Anaesthesiology, GSL Medical College, Rajahmundry.

4. Professor \& HOD. Department of Anaesthesiology, GSL Medical College, Rajahmundry.

5. Post Graduate. Department of Anaesthesiology, GSL Medical College, Rajahmundry.

\section{CORRESPONDING AUTHOR:}

Dr. S. Kartika Balaji,

Department of Anaesthesiology and Critical Care,

GSL Medical College,

Lakshmipuram, Rajahmundry- 533296,

Andhra Pradesh.

E-mail: kartik1414@gmail.com

ABSTRACT: Here, we report a fifty six year old male patient with intrathoracic incarceration and perforation of the stomach on the left side of the diaphragmatic defect resulting as empyema and a late sequelae of barium induced pleural thickening where decortication was performed. It is not usual, without a history of trauma patient developing acquired Bochdalek hernia with empyema as seen in our case. Anaesthetic management was successful in the repair of acquired Bochdalek hernia during the first surgery and decortication of the lung during the second surgery in our patient.

KEYWORDS: Diaphragmatic hernia, Bochdalek hernia, Barium, Pleural thickening, Decortication.

INTRODUCTION: Bochdalek hernia $(\mathrm{BH})$ is a type of congenital diaphragmatic hernia seen primarily in children. It is rare in elderly accounting for $0.17-6 \%$ of all diaphragmatic hernias. $[1,2]$ The majority present during neonatal life with congenital pulmonary abnormalities and have poor prognosis. Adult BH is rare, remain largely asymptomatic and its clinical presentation is varied with respiratory or gastrointestinal systems involved primarily. Chest radiographs (CXR), Computerised Tomography (CT) plays an important role in diagnosing and assessing the severity. ${ }^{[3,4]}$

Here we report a rare case of Left sided BH in adult which caused pyopneumothorax and also pleural thickening, a late complication due to barium from intrathoracic incarceration and perforation of the stomach (fundus).

CASE REPORT: A fifty six year old male presented with acute onset of localised left sided chest pain with dyspnea grade III (American Heart Association), high grade fever associated with chills, rigors, discharging sinuses on the left fifth intercostals space of mid axilla. The patient illness evaluation dates back to eight months where his clinical findings along with an emergency CXR gave a provisional diagnosis of left hydro/pyopneumothorax in shock. [Figure 1] Intercostal chest tube drain (ICD) was inserted and the patient was fine until dramatic increase in fluid level inside the water seal. Patient was allowed to take oral feeds the following day where in with each oral feed, food particles were draining through the chest tube. Further 
evaluation with Barium contrast, upper gastrointestinal endoscopy, CT scan revealed BH with fundus of stomach, spleen in the thorax. [Figure 2] Intrathoracic incarceration and perforation of the fundus (stomach) resulted in left pyopneumothorax for which surgical correction of BH was done. The present clinical scenario of the patient with loculated pyothorax and pleural thickening [Figure 3] resulted due to Barium leak into the pleura, empyema for which decortication procedure was planned.

Patient's preoperative cardiac evaluation with electrocardiograph (ECG) was within normal limits, 2D Echo was normal with no significant abnormality and an ejection fraction of $65 \%$. Blood urea was $41 \mathrm{mg} / \mathrm{dl}$ and serum creatinine $1.0 \mathrm{mg} / \mathrm{dl}$. Differential count showed neutrophils $60 \%$, lymphocytes $25 \%$, eosinophils $13 \%$, monocytes $2 \%$ and White blood cells $18,800 / \mathrm{mm}^{3}$. Patient's pulmonary function test showed reduction in forced vital capacity (FVC) and forced expiratory volume in one second (FEV1).

Anaesthetic management was done with preoperative stabilisation of the patient with broad antibiotic Amoxiclav 625mg TID, steam inhalations, nebulisation with salbutamol and budesonide, chest physiotherapy, breathing exercises and posted for decortication. Intraoperative monitoring with Electrocardiography, Non-invasive blood pressure, Pulse oximetry, Capnography was performed. Premedication with intravenous (IV) Midazolam 3mg,

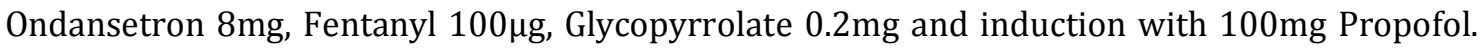
Non-depolarising muscle relaxant Vecuronium $5 \mathrm{mg}$ given for paralyzing and intubated with 37Fr left-sided double lumen tube (DLT). DLT position was checked by blocking tracheal and bronchial tubes, and then fixed after confirmation of it in place. Oxygen: Nitrous oxide kept at 1:2 litres/minute initially and patient kept in right lateral position. Later on, only oxygen at 2litres/minute was given. Decortication procedure was performed and ICD placed. Pleura of 6.5 $\times 4.5 \times 2 \mathrm{cms}$ were removed. Patient was extubated with Neostigmine $2.5 \mathrm{mg}$, Glycopyrrolate $0.5 \mathrm{mg}$ and shifted to intensive care unit. Intercostal nerve block was given by the surgeon at the end of surgery for post operative analgesia and later on maintained with drugs like fentanyl, tramadol and paracetomol IV. Patient was positioned in the lateral decubitus position with the affected side up. Patient's recovery was good and discharged on fifth day after ICD removal. Incentive spirometry and breathing exercises were done by the patient for the improvement of lung function post surgery.

DISCUSSION: Congenital diaphragmatic hernias occur through an anterior parasternal foramen (Morgagni) or through a posterolateral, mainly left-sided defect (Bochdalek) representing persistence of the pleuroperitoneal canal. Adult BHs present with gastrointestinal (epigastric pain, dyspepsia, nausea, vomiting) or respiratory (dyspnea, wheezing) symptoms followed by severe attacks, episodes of incarceration with serious consequences. Symptoms can be intermittent causing regression when herniated viscera spontaneously reduce or severe associated with strangulation of herniated viscera when the diagnosis has been missed. The organs most commonly herniated into thorax are stomach, ileum, colon and spleen. In our case, herniation of stomach along with incarceration is seen. The liver may herniate along with the bowel loops if defect is on the right side. ${ }^{5]}$ Though rare, gastric volvulus is one of the recognised complications of $\mathrm{BH}$. ${ }^{[6]}$

Barium sulphate is insoluble salt of barium used as radiographic contrast medium. Barium swallow is the most common routine procedure in the examination of oropharynx, oesophagus and upper gastrointestinal system. ${ }^{[7]}$ Increased fluid level in the underwater seal along with the food particles made the surgeons go for fluoroscopy with barium meal to know 
whether there is a communication between enteron to pleural space or enteron to ICD (iatrogenic). Fluoroscopy revealed intrathoracic incarceration and perforation of stomach (fundus) which led to leakage of barium into the pleura causing pleural thickening in our case. It is not usual barium leaking from ruptured stomach into the pleural cavity, first in the literature though there are reported cases of barium aspiration leading to complications. [8]

Thoracic empyema is a serious problem which usually results from pulmonary infection or thoracic surgery. Treatment includes sepsis control with antibiotics, restoration of pulmonary function and prevents lung entrapment after peeling. Surgical techniques to drain the pleural fluid, clearing peel from the lung, obliterating the empyema space are often required. Majority of the patients were in exudative state followed by fibrinopurulent and organised state. These procedures have in fact relieved a renaissance in recent years due to an increase of complex intra-thoracic diseases. Some patients present with sepsis at the time of the surgery in whom placement of a thoracic epidural catheter is not recommended. These patients require lung isolation preferably with a DLT as in our case. DLT facilitates suction of debris, copious secretions present in the tracheobronchial tree. [9]

Decortication removes a restrictive layer of fibrous tissue overlying the lung, chest wall and diaphragm. Presence of symptoms due to lung restriction resulting from the development of thick peel as in our case due to barium is the indication.[10] Perioperative work up includes thorough history, pulmonary function tests, spirometry, blood gas, cardiopulmonary exercise test to avoid unnecessary complications. Assessing cardiac risk factors in patients undergoing decortications is important as they have highest incidence of associated postoperative congestive heart failure, arrhythmias and myocardial reinfarction. [9]

Patients undergoing decortications may have massive blood loss if the lung has been chronically collapsed. Expansion should be done gradually to avoid the development of pulmonary edema on reexpansion. Assessing gas exchange, impending respiratory failure and ability to protect airways help in taking a decision to initiate or continue mechanical ventilation. Majority of the patients can be extubated in the operating room and is encouraged if they meet standard criteria. Complications associated with decortications are perioperative sepsis syndrome, broncho-pleural fistulas, bleeding, persistent air leaks. Second surgery may be required in cases of prolonged air leaks and bronchopleural fistulas. ${ }^{9,11]}$

\section{REFERENCES:}

1. Mark E, Jeffrey SS, Saini SS, Peter RM. Prevalence of incidental Bochdalek's hernia in a large adult population. AJR 2001; 177:363-66.

2. Gale ME. Bochdalek hernia: prevalence and CT characteristics. Radiology 1985; 156:44952.

3. Nouheim KS. Adult presentation of unusual diaphragmatic hernias. Chest Surg Clin N Amer 1998;8:359-69.

4. Wiseman NE, MacPherson RI. "Acquired "congenital diaphragmatic hernia. J Paediatr Surg 1997; 12:657.

5. Giannoulis K, Sutton R. Bochdalek hernia presenting in adult life: report of an unusual case and review of the literature. Annals of Gastroenterology 2004; 17:109-12.

6. Perhoniemi V, Helminen J, Luosto R. Posterolateral diaphragmatic hernia in adults. Acute symptoms, diagnosis and treatment. Scand J Thorac Cardiovasc Surg 1992;26:225-27. 
7. Zaino C, Beneventano TC. Radiologic examination of the oropharynx and esophagus. New York: Springer; 1977, pp 1-28.

8. Pracy JPM, Montgomery PQ, Reading N. Acute pneumonitis caused by low-density barium sulphate aspiration. J Laryngol Otol 1993;1 07:347-8.

9. Peter D. Slinger, Javier H. Campos. Anesthesia for thoracic surgery. In: Ronald D. Miller,

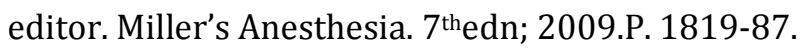

10. Colice GL, Curtis A, Deslauriers J, Heffner J, Light R, Littenberg B et al. Medical and surgical treatment of parapneumonic effusions: an evidence- based guideline. Chest 2000; 118:1158-71.

11. Thomas W. Rice. Fibrothorax and decortication of the Lung. In: Thomas W. Shields, Joseph Locicero, Carolyn E. Reed, Richard H. Feins editors. General Thoracic Surgery

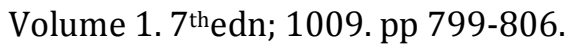

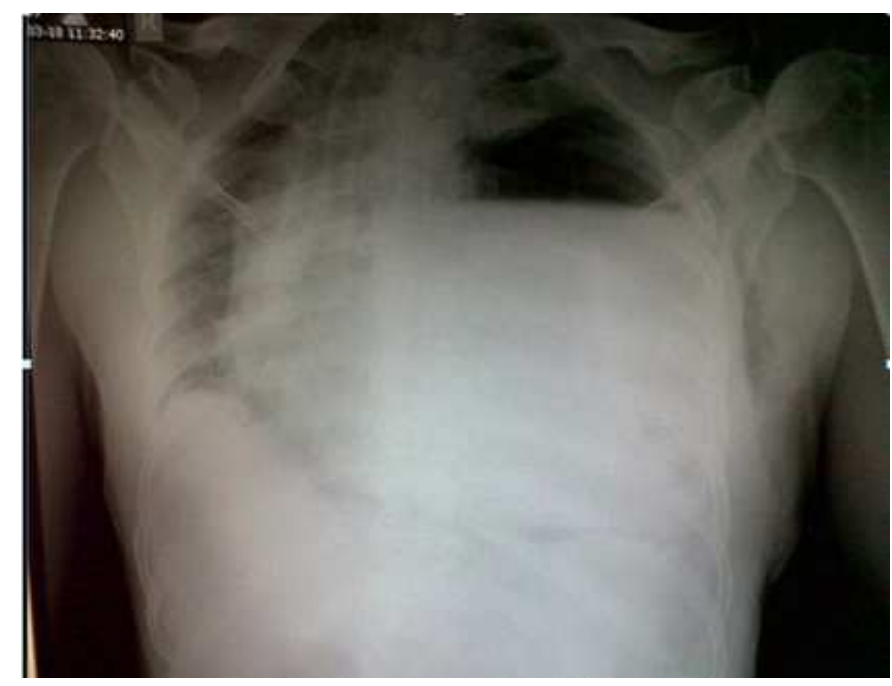

Hydropyopneumothorax

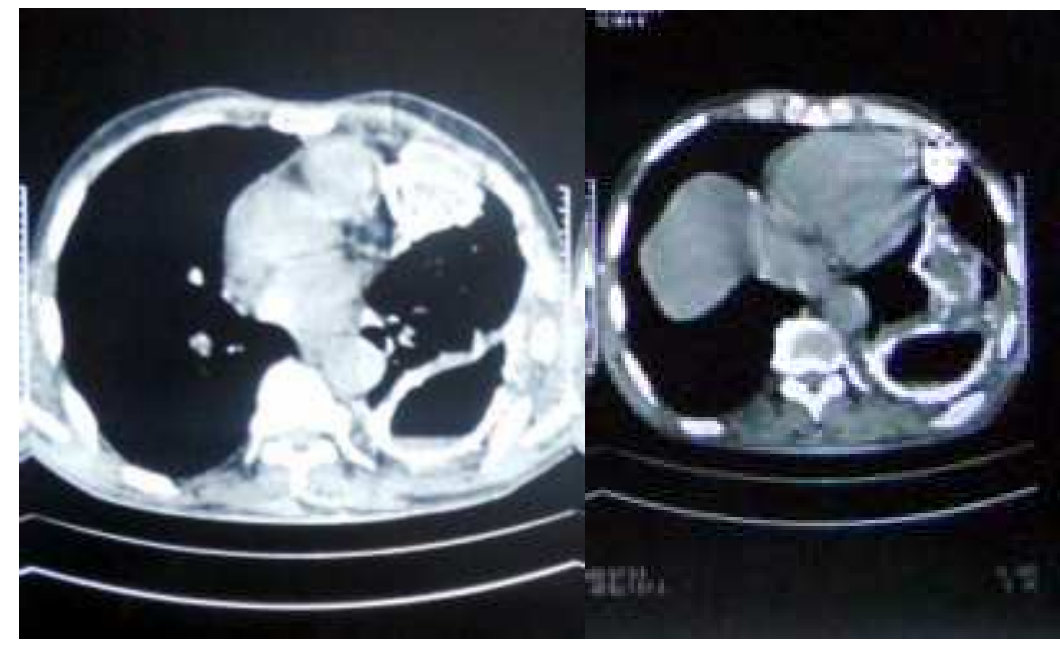

Pleural thickening

Stomach fundus and spleen in the left hemithorax 